\title{
PROPER MAPPINGS AND DIMENSION
}

\section{JAMES KEESLING}

AbStract. In this note it is shown that if $W$ is the long line and $f$ is a proper mapping of $W \times[0,1]$ into $Y$, then $\operatorname{dim} Y \geqq 2$. This answers a question raised by Isbell.

The main result of this note is that if $W$ is the long line and $f$ is a proper mapping of $W \times[0,1)$ into $Y$, then $\operatorname{dim} Y \geqq 2$. This answers a question posed by Isbell [2, pp. 119-120]. The main result will follow after a few preliminary results.

Lemma. Let $X=[0,1] \times[0,1)$ with $C_{0}=\{0\} \times[0,1)$ and $C_{1}=\{1\}$ $\times[0,1)$. If $D$ is a closed set which separates $C_{0}$ and $C_{1}$ in $X$, then $D$ has a noncompact component.

Proof. Suppose that $X-D=U \cup V$ is a separation with $C_{0} \subset U$ and $C_{1} \subset V$. There is a continuous $f: U \cup D \rightarrow[0,1]$ which is 0 on $C_{0}$ and 1 on $D$. There is a continuous function $g: V \cup D \rightarrow[1,2]$ which is 1 on $D$ and 2 on $C_{1}$. Thus there is a continuous function $h: X$ $\rightarrow[0,2]$ which is 0 on $C_{0}, 1$ on $D$, and 2 on $C_{1}$. Accordingly there is a homeomorphism $i: X \rightarrow X$ taking $C_{0}$ to $C_{0}, C_{1}$ to $C_{1}$, and $D$ to a set $E$ whose closure in $R^{2}$ is disjoint from $\bar{C}_{0}$ and $\bar{C}_{1}$. Then $\bar{E}$ separates the two sides of $[0,1] \times[0,1]$ and thus one of its components $K$ intersects both the top and bottom of the square. Now $K$ contains a component of $E$ and it cannot contain a compact component of $E$. Therefore $E$ and thus $D$ have noncompact components.

Proposition. Let $X, C_{0}$, and $C_{1}$ be as in the Lemma. If $f(X)=Y$ is a proper mapping with $f\left(C_{0}\right) \cap f\left(C_{1}\right)=\varnothing$, then $\operatorname{dim} Y \geqq 2$.

Proof. We note that $Y$ is separable metric by [6]. Thus the various dimension functions coincide as proved in [1]. We will show that Ind $Y \geqq 2$ by showing that $f\left(C_{0}\right)$ and $f\left(C_{1}\right)$ cannot be separated in $Y$ by a closed 0 -dimensional set. Suppose that $E$ is closed and separates $f\left(C_{0}\right)$ and $f\left(C_{1}\right)$ in $Y$. Then $f^{-1}(E)$ is closed in $X$ and separates $C_{0}$ and $C_{1}$. By the Lemma, $f^{-1}(E)$ has a noncompact component $D$. Since $f$ is proper and $D$ is closed, $f$ cannot be constant on $D$. Therefore $f(D)$ is connected and not a point and thus $\operatorname{dim} f(D) \geqq 1$ and $\operatorname{dim} E \geqq 1$. This proves the Proposition.

Received by the editors July 22, 1970.

AMS 1970 subject classifications. Primary 54F45; Secondary 54C10.

Key words and phrases. Dimension, proper mapping, locally compact space. 
TheOREM. If $f: W \times[0,1) \rightarrow Y$ is proper, then $Y$ contains a compact metric subspace $D$ with $\operatorname{dim} D \geqq 2$.

Proof. Let $A_{0}=\{0\} \times\left[0,1-2^{-1}\right]$. Using the fact that $f^{-1} f\left(A_{0}\right)$ is compact, let $A_{1}=\left[0, \alpha_{1}\right] \times\left[0,1-2^{-n_{1}}\right]$ be such that $f^{-1} f\left(A_{0}\right) \subset$ int $A_{1}$. Continue inductively defining $A_{i}=\left[0, \alpha_{i}\right] \times\left[0,1-2^{-n_{i}}\right]$ such that $f^{-1} f\left(A_{i}\right) \subset$ int $A_{i+1}$ for all $i$. Let $B=\bigcup_{i=1}^{\infty} A_{i}=\bigcup_{i=1}^{\infty} f^{-1} f\left(A_{i}\right)$. Then $B$ is an inverse set and $B=[0, \gamma) \times[0,1)$ where $\gamma=\sup \left\{\alpha_{i}\right\}=\lim \alpha_{i}$. Now $\bar{B}=[0, \gamma] \times[0,1)$ and $f \mid \bar{B}$ is proper. Letting $C_{0}=\{0\} \times[0,1)$ and $C_{1}=\{\gamma\} \times[0,1)$ we have that $f\left(C_{0}\right) \cap f\left(C_{1}\right)=\varnothing$. Applying the Proposition we get that $\operatorname{dim} f(\bar{B}) \geqq 2$. Since $f(\bar{B})$ is separable metric and locally compact it contains a compact metric $D$ with $\operatorname{dim} D \geqq 2$.

CoROllary. There is a space $Y$ which has the property that $\min \operatorname{dim}(Y-\{p\})=\min \operatorname{dim} Y-2$ for some point $p \in Y$.

An outline of the proof of the Corollary assuming the Theorem is given on p. 120 of [2]. There is no need to elaborate here.

REMARK. The cited proof outline of Isbell to the Corollary requires a space $X$ which is locally compact and has the property proved for $W \times[0,1)$ in the Theorem of this paper. Such a space cannot be paracompact (and consequently not metric) since such a space would be the free union of locally compact Lindelöf spaces $S_{\alpha}$. Each $S_{\alpha}$ admits a proper mapping into $[0, \infty)$ and thus the free union of the $S_{\alpha}$ 's admits a proper mapping in to the free union of an appropriate collection of half-lines.

If local compactness were not needed, then there are separable metric spaces satisfying this condition. Take for example any separable metric space $X$ which is totally disconnected and $n$-dimensional. Such spaces exist for every $n \geqq 1[3$, p. 152]. In [4] such spaces are constructed which are also complete. For such a space $X$, if $f(X)=Y$ is proper, then $Y$ is separable metric by [6]. Also for each $y \in Y$, $\operatorname{dim} f^{-1}(y)=0$, since $f^{-1}(y)$ is compact and totally disconnected [1, p. $22]$. By the Hurewicz theorem for closed mappings which lower dimension $[1$, p. 91], we must have $\operatorname{dim} Y \geqq \operatorname{dim} X=n$. That is, the dimension of such a space cannot be lowered by a proper mapping.

\section{REFERENCES}

1. W. Hurewicz and H. Wallman, Dimension theory, Princeton Math. Series, vol. 4, Princeton Univ. Press, Princeton, N.J., 1941. MR 3, 312.

2. J. Isbell, Uniform spaces, Math. Surveys, no. 12, Amer. Math. Soc., Providence, R.I., 1964. MR $30 \# 561$.

3. K. Kuratowski, Topologie. Vol. 2, Monografie Math., Tom 21, PWN, Warsaw, 
1961; English transl., Academic Press, New York; PWN, Warsaw, 1968. MR 24 \#A2958.

4. A. Lelek, Some problems in metric topology, Mimeographed Lecture Notes, Louisiana State University, Baton Rouge, La., 1965/66.

5. J. Nagata, Modern dimension theory, Bibliotheca Math., vol. 6, Interscience, New York, 1965. MR 34 \#8380.

6. A. H. Stone, Metrizability of decomposition spaces, Proc. Amer. Math. Soc. 7 (1956), 690-700. MR 19, 299.

University of Florida, Gainesville, Florida 32601 